\title{
Depression comorbidity in chronic tension-type headache: Own experience and literature review
}

\author{
Domenico Chirchiglia', Pasquale Chirchiglia', Rosa Marotta² \\ ${ }^{1}$ Department of Neurosurgery, University of Catanzaro, Campus Germaneto, VLE Europa, Catanzaro, Italy \\ ${ }^{2}$ Department of Health Sciences, University of Catanzaro, Campus Germaneto, \\ VLE Europa, Catanzaro, Italy
}

\begin{abstract}
Comorbid depression in tension-type headache is a condition well known. A link correlates the two disorders but the mechanism is not perfectly clear. The purpose of this work is to give our experience on the subject and to compare it with the literature data.

We investigated a number of cases of tension-type headache patients, with the aim of finding signs of depression as comorbidity.

The results showed a depression comorbidity in tension-type headache, with a $67 \%$ percentage, in line with the literature data.

There is evidence of comorbidity of depression in tension-type headache. The mechanisms that bind the two disorders appear to be explained by the activity of neurotransmitters common to both.
\end{abstract}

Keywords: headache, tension-type headache, depression

\section{INTRODUCTION}

Tension-type headache (TTH) is a primary headache, once known as psychogenic headache. According to International Headache Society (IHS), it was codified as 2, while the chronic form as 2.3. (Chronic tension-type headache). TTH is very frequent, between 30 and $78 \%$. It may be episodic and chronic, associated with or not contracture of the pericranial muscles. Then,episodic sporadic TTH: at least 10 episodes occurring for less than 12 days per year (therefore less than one episode per month), and episodic frequent TTH: at least 10 episodes occurring for 1-14 days a month for at least 3 months.

Episodic forms can evolve into chronic form. The chronic form is manifested by daily or very frequent episodes of headache (more than 15 days per month for more than 3 months). It seems that the underlying pain mechanism in the chronic TTH is central (1). Pain is bilateral, pressing or lightening quality, mild or moderate, duration hours to days and can associated with nausea, photophobia,phonofobia,osmofobia. Comorbidity between TTH and psychiatric disorders such as anxiety and depression is known. Sometimes a depression does not manifest with mood variation, but with somatoform symptoms, including headaches.

Depression is more prevalent in headache patients than in the headache-free population. Epidemiological studies suggest a common genetic, biochemical or environmental factor between primary headaches and depression. Comorbidity is supported by the similar concentration of neurotransmitters in both disorders, TTH and depression (2).

In fact, a theory calls into question a reduction of serotonin levels: the administration of tricyclic 
antidepressants such as amitriptyline has been successfully used in the treatment of TTH, although the results obtained probably depend not only on serotonin reuptake inhibition but also on other mechanisms. Serotoninergic pathways are altered in depression, and serotonin reduction in synapses of depressed subjects has been detected, as well as in TTH subjects. The old term of psychogenic headache, attributed to TTH, stems from the changes in neurotransmitters, as well as associated mood disorders, often found in this form of headache.

\section{MATERIAL AND METHODS}

We have considered 30 cases of chronic TTH, observed over a period of two years. The sample consisted of 15 males and 15 females, aged between 30 and 50 years old, average 38,86. All presented pressing and bilateral pain, an average of 2 attacks per month, duration several hours, phono-photophobia, nausea. They all had taken non-steroidal anti-inflammatory drugs (NSAIDs) that were not always effective, as evidenced by the administration of the Visual Analogue Scale (VAS) . Fourtheen patients assumed antidepressans which as it is known have been shown useful in TTH. Twenty of them (67\%) lamented anxiety, lack of pleasure, fatigue, crying crises. All were tested for depression through the administration of the Hamilton Depression Rating Scale (HDRS). HDRS is designed to rate the severity of depression in patients. Although it contains 21 areas, the patient's score is calculated on the first 17 answers.Scoring is based on the 17-item scale and scores of 0-7 are considered as being normal, 8-16 suggest mild depression, 17-23 moderate depression and scores over 24 are indicative of severe depression; the maximum score being 52 on the 17-point scale. The

TABLE SAMPLE

\begin{tabular}{|c|c|c|c|c|c|}
\hline Age-Sex & TTH-Therapy VAS & $\begin{array}{c}\text { Depression HDRS/Therapy/ } \\
\text { Before }\end{array}$ & $\begin{array}{c}\text { Depression Therapy } \\
\text { Modified } \\
\end{array}$ & Results TTH-VAS & $\begin{array}{c}\text { Results } \\
\text { Depression }\end{array}$ \\
\hline 1) $35-M$ & NSAIDs 6 & 18/ amitriptyline & paroxe ne $10 / \mathrm{mg}$ & 2/ improved & disappearance \\
\hline 2) $47-M$ & NSAIDs 7 & 8/no therapy & paroxe ne $10 / \mathrm{mg}$ & 3/ improved & disappearance \\
\hline 3) $50-M$ & NSAIDs 7 & 16/ amitriptyline & paroxe ne $10 / \mathrm{mg}$ & 3/ improved & disappearance \\
\hline 4) $45-M$ & NSAIDs 8 & $11 /$ amitriptyline & paroxe ne $10 / \mathrm{mg}$ & 4/ improved & disappearance \\
\hline 5) $30-M$ & NSAIDs 8 & 10/ no therapy & paroxe ne $20 / \mathrm{mg}$ & 4/ improved & disappearance \\
\hline 6) $42-M$ & NSAIDs 7 & 15/ amitriptyline & paroxe ne $10 / \mathrm{mg}$ & 3/ improved & disappearance \\
\hline 7) 49 - M & NSAIDs 8 & 16/ amitriptyline & paroxe ne $20 / \mathrm{mg}$ & 3/ improved & disappearance \\
\hline 8) $45-M$ & NSAIDs 8 & 14/ amitriptyline & paroxe ne $20 / \mathrm{mg}$ & 4/ improved & disappearance \\
\hline 9) 48 - M & NSAIDs 7 & 14/ amitriptyline & paroxe ne $10 / \mathrm{mg}$ & 4/ improved & disappearance \\
\hline 10) $32-M$ & NSAIDs 8 & 16/ amitriptyline & paroxe ne $20 / \mathrm{mg}$ & 4/ improved & disappearance \\
\hline 11) $50-M$ & NSAIDs 7 & 14/ no therapy & paroxe ne10/mg & 2/ improved & disappearance \\
\hline 12) $36-M$ & NSAIDs 4 & absent & no therapy & not improved & \\
\hline 13) $45-M$ & NSAIDs 5 & absent & no therapy & not improved & \\
\hline 14) $37-M$ & NSAIDs 4 & absent & no therapy & not improved & \\
\hline 15) $42-M$ & NSAIDs 3 & absent & no therapy & not improved & \\
\hline 16) $35-F$ & NSAIDs 4 & absent & no therapy & not improved & \\
\hline 17) $42-F$ & NSAIDs 3 & absent & no therapy & not improved & \\
\hline 18) $37-F$ & NSAIDs 4 & absent & no therapy & not improved & \\
\hline 19) $50-F$ & NSAIDs 3 & absent & no therapy & not improved & \\
\hline 20) $45-F$ & NSAIDs 3 & absent & no therapy & not improved & \\
\hline 21) $37-F$ & NSAIDs 4 & absent & no therapy & not improved & \\
\hline 22) $30-F$ & NSAIDs 7 & $16 /$ no therapy & paroxe ne $20 / \mathrm{mg}$ & 3/ improved & disappearance \\
\hline 23) $48-F$ & NSAIDs 7 & 16/ amitriptyline & paroxe ne $20 / \mathrm{mg}$ & 3/ improved & disappearance \\
\hline 24) $35-F$ & NSAIDs 8 & $17 /$ amitriptyline & paroxe ne $20 / \mathrm{mg}$ & 4/ improved & disappearance \\
\hline 25) $42-F$ & NSAIDs 7 & 16/ amitriptyline & paroxe ne $20 / \mathrm{mg}$ & 3/ improved & disappearance \\
\hline 26) $48-F$ & NSAIDs 7 & $15 /$ no therapy & paroxe ne $20 / \mathrm{mg}$ & 2/ improved & disappearance \\
\hline 27) $39-F$ & NSAIDs 7 & $16 /$ no therapy & paroxe ne $20 / \mathrm{mg}$ & 2/ improved & disappearance \\
\hline 28) $48-F$ & NSAIDs 7 & 15/ amitriptyline & paroxe ne $20 / \mathrm{mg}$ & 2/ improved & disappearance \\
\hline 29) $42-F$ & NSAIDs 8 & 16/ amitriptyline & paroxe ne $20 / \mathrm{mg}$ & 3/ improved & disappearance \\
\hline 30) $37-F$ & NSAIDs 8 & 16/ amitriptyline & paroxe ne $20 / \mathrm{mg}$ & 3/ improved & disappearance \\
\hline
\end{tabular}


results were positive for the 20 subjects who had shown signs of depression. Therefore, we administered paroxetine 10 or $20 \mathrm{mg}$ oral daily dose, depending on the severity of the symptoms. The results were the following: the 20 patients with TTH associated with depression showed a strong improvement both for the depressive symptoms and the headache. At present, after two years of control, depressive symptoms have disappeared, while headache symptomatology has improved, both for frequency and intensity.

\section{DISCUSSION}

There is comorbidity between depression and tension-type headache (TTH). Literature data confirm this correlation. Psychological factors and emotional disorders have been indicated as risk factors for TTH (3). Episodic headache of the tension type is the most prevalent primary headache with a lifetime prevalence of about $78 \%$. Clinical characteristics are a dull, moderate, diffusedheadache without accompanying autonomic or vegetative symptoms. The episodic tension-type headache often lasts only 30 min up to a maximum of a few days. In contrast to this clinically often undemanding headache, chronic tension-type headache can cause considerable disability in patients. The 1-year prevalence is $1-3 \%$ of the population. All therapy strategies combine nonpharmaceutical therapy such as psychological treatment with pharmaceutical treatment such as tricyclic antidepressants or combined serotoninergic and noradrenergic antidepressants. Combination therapy has been proven to be more effective than singular strategies.Episodic tension-type headache is a common problem affecting approximately $2-3 \%$ of the population $(2,3)$. The origin of tension-type headache is multifactorial, but the pathogenesis is still unclear. In some individuals episodic tension-type headache transforms into chronic tension-type headache (cTTH). Tension-type headache (TTH) is the most common form of headache, and chronic tension-type headache (cTTH) is one of the most neglected and difficult types of headache to treat. The pathogenesis of TTH seems to be related to peripheral mechanisms as myofascial nociception and central mechanisms as sensitisation and inadequate endogenous pain control. Acute therapy is effective for episodes of
TTH, whereas preventive treatment, which is indicated for frequent and chronic TTH is, on average, not effective. For most patients with cTTH, the combination of drug therapies and non-drug therapies (such as relaxation and stress management techniques or physical therapies) is recommended. There is evidence suggesting that subjects with TTH exhibit co-morbid anxiety, depression, or sleep disturbances. Some studies have previously investigated the association between depression andheadache. Zebenholzer et al. observed that coexistence of depression and anxiety had a significant impact in patients with TTH. It would be conceivable that different variables can interact at different levels to headache associated to depression (4). It seems that emotional or stressful factors are common precipitating factors of headache episodes in patients with TTH, since they may trigger hyperalgesic responses within the central nervous system. Therefore, it is possible that emotional factors can trigger more headache attacks inducing an increasing in the frequency of headaches. Then, the presence of mood disorders is more associated to the frequency of headaches. Since emotional stress is the most common trigger for pain in subjects with TTH, proper management of emotional factors may be relevant for avoiding chronification. In fact, emotional stress and depression represent two modifiable risk factors implicated in the progression from episodic to chronic headaches.Current findings would suggest that management of patients with cCTTH should include therapeutic interventions as cognitive behavioral techniques to decrease depressive symptoms. In a study conducted by Matta, among the patients with chronic TTH, depression was observed in $40 \%$ (4). Serrano Duena found in patients with chronic TTH indices of depression in $33.7 \%$ of cases (5). But what is the mechanism that binds depression and TTH? One response is given by the medications that are used as effective in both conditions. Tricyclic antidepressants as well as SSRIs are the drugs widely used in depressive syndromes, but also in TTH (79). This is explained by alteration of neurotransmitters that don't well circulate in the synaptic terminations of depressed subjects as well as in patients with TTH. It is also true that antidepressants are not always effective in TTH $(10,11)$. This creates some doubts about the link between two disorders. An- 
other response is given by two symptoms present both in depression with strong anxious component, and in TTH. They are osmophobia and phonophobia $(6,12,13)$.Regarding mechanism linking osmophobia and phonophobia with TTH, osmophobia, phonophobia and psychiatric disorders might possibly result from dysfunction of common brain structures, such as the insular cortex, hippocampus or amygdala which are related with emotions (14). The pathophysiological mechanisms we have highlighted evoke the old synonym " psychogenic headache" for TTH. In short, a link between the two disorders concerns the emotional sphere and the neurotransmitters involved in the limbic circuits $(15,16)$. These are suggestive assumptions that lead to a least partial explanation of this comorbidity.

\section{CONCLUSIONS}

TTHs are very common in general population and may be under diagnosed. They are more com- mon than patients suffering from migraine headaches. In general underlying psychiatric conditions, such as anxiety and depression, are prevalent in TTH sufferers. For acute therapy NSAIDs are the main treatment option. Tricyclic antidepressant, SSRI, and cognitive behavioral therapy have been proven to be effective for prophylactic purposes.

There is a comorbidity between depression and tension-type headache. The data we have obtained from the literature are strongly suggestive for a close relationship between the two disorders. Our experience showed a depression comorbidity in TTH of $67 \%$, a substantial percentage and in line with the literature data. TTH is a frequent form of headache, with "psychogenic characteristics", similar to the symptoms of depression. We believe that alterations of neurotransmission and dysfunctions of the emotional sphere are the link between TTH and depression.

Conflict of interest: none declared Financial support: none declared

\section{REFERENCES}

1. International Classification Headache Disorders, 3 beta version, 2018

2. Torelli P., Abrignani G., Castellini P., Lambru G., Manzoni G.C. Human psyche and tension-type headache. Neurol sci 2008 May; 29 Suppl 1:S93-5. doi: 10.1007/s10072-008-0896-3.

3. Peñacoba-Puente C., Fernández-de-Las-Peñas C., González-Gutierrez J.L., Miangolarra-Page J.C., Pareja J.A. Interaction between anxiety, depression, quality of life and clinical parameters in chronic tension-type headache. Eur J Pain. 2008 Oct; 12(7):886-94. doi: 10.1016/j.ejpain.2007.12.016. Epub 2008 Mar 10.

4. Matta A.P., Moreira Filho P.F. Depressive symptoms and anxiety in patients with chronic and episodic tension-type headache]. Arq Neuropsiquiatr. 2003 Dec; 61(4):991-4. Epub 2004 Jan 6. J Neurol Sci.

5. Serrano-Dueñas M. Chronic tension-type headache and depression Rev Neurol. 2000 May 1-15;30(9):822-6.

6. Silva-Néto R.P., Peres M.F., Valença M.M. Accuracy of osmophobia in the differential diagnosis between migraine and tension-type headache. Neurol sci 2014 Apr 15; 339(1-2):118-22. doi: 10.1016/j. jns.2014.01.040. Epub 2014 Feb 6.

7. Jensen P. Pathophysiological mechanisms of tension-type headache: a review of epidemiological and experimental studi Cephalalgia. $1999 \mathrm{Jul} ; 19(6): 602-21$.

8. Diaz-Insa S. The myth of tension-type headache. Rev Neurol. 2014 Mar 10; 58 Suppl 2:S27-31.

9. Probyn K., Bowers H., Caldwell F., Mistry D., Underwood M., Matharu M., Pincus T. CHESS Team. Prognostic factors for chronic headache: A systematic review. Neurology. 2017 Jul 18; 89(3):291301. doi: 10.1212/WNL.0000000000004112. Epub 2017 Jun 14.

10. Gesztelyi G. Primary headache and depression. Orv Hetil. 2004 Nov 28; 145(48):2419-24.

11. Rasmussen B.K., Jensen R., Schroll M., Olesen J. Epidemiology of headache in a general population--a prevalence study. J Clin Epidemiol. 1991; 44(11):1147-1157. doi: 10.1016/08954356(91)90147-2. 0.

12. Lyngberg A.C., Rasmussen B.K., Jorgensen T., Jensen R. Has the prevalence of migraine and tension-type headache changed over a 12-year period? A Danish population survey. Eur J Epidemiol. 2005; 20(3):243-249. doi: 10.1007/s10654-004-6519-2. 31.

13. Krogh A.B., Larsson B., Linde M. (2015) Prevalence and disability of headache among Norwegian adolescents: A cross-sectional school-based study. Cephalalgia. doi:10.1177/0333102415573512

14. Varkey E., Hagen K., Zwart J.A., Linde M. Physical activity and headache: results from the Nord-Trondelag Health Study (HUNT) Cephalalgia. 2008; 28(12):1292-1297. doi: 10.1111/j.1468-2982.2008.01678.x.

15. Westergaard M.L., Hansen E.H., Glumer C., Olesen J., Jensen R.H. Definitions of medication-overuse headache in population-based studies and their implications on prevalence estimates: a systematic review. Cephalalgia. 2014; 34(6):409-425. doi: 10.1177/0333102413512033.

16. Uprety Y., Asselin H., Boon E.K., Yadav S., Shrestha K.K. Indigenous use and bio-efficacy of medicinal plants in the Rasuwa District, Central Nepal. J Ethnobiol Ethnomed. 2010; 6:3. doi: 10.1186/1746-4269-6-3. 